\title{
Synthesis, Identification and Biological Activity of Some New Chalcone derivatives from 8-Chlorotheophylline
}

\author{
NABEEL A. ABDULREDA and SALWA R. ABDUL-AMEER* \\ ${ }^{1}$ Department of Chemistry, College of Science, University of Al-Qadisiyah, Iraq. \\ 2Department of Chemistry, College of Education University of Al-Qadisiyah, Iraq. \\ Corresponding author E-mail: salwa22.razzaq@gmail.com
}

http://dx.doi.org/10.13005/ojc/340144

(Received: September 25, 2017; Accepted: December 20, 2017)

\begin{abstract}
New derivatives of 8-Chloro-theophylline (8-CTh) were synthesized by react 8-CTh and 4-amino acetophenone to prepared 8-(4-acetylphenylamino)-1,3-dimethyl-1H-purine-2,6(3H,7H)dione then the result reacts with some substituted benzaldehyde derivatives to prepared different chalcone compound then the products were allowed to react with thiourea and hydrazine to give Pyrimidine and pyrazoline derivatives respectively. The reaction was monitored by thin layer chromatography (TLC) technique. All new compounds were characterized by melting points, elemental analysis, FTIR, ${ }^{1} \mathrm{H},{ }^{13} \mathrm{C}$ and 2D NMR spectroscopy. Antimicrobial activity of these derivatives was also determined.
\end{abstract}

Keywords: 8-Chlorotheophylline, Chalcone, Pyrazoline, Pyrimidine, Antimicrobial.

\section{INTRODUCTION}

Xanthine a purine base produces in most human body tissues and fluids and in other organisms it is produced by purine metabolism. The commonly used methyl xanthine theophylline, caffeine and theobromine are naturally occurring compounds in various plants. ${ }^{2}$ These substances are components of most widely consumed beverages, coffee, tea and cocoa. Theophylline and its salts are used in the therapy of bronchial asthma and chronic obstructive pulmonary disease (COPD) $8-C T h$ is a purine derivative which is obtained from theophylline ${ }^{3}$.

In therapy, 8-CTh is used in combination with antihistamines in order to enhance their action against travel sickness and other hearing labyrinth diseases ${ }^{4}$. In this work prepared some derivatives from 8-CTh by chalcone reaction. Chalcone can be prepared by condensation reaction between different aldehyde compound and an

This is an Open Access article licensed under a Creative Commons Attribution-NonCommercial-ShareAlike 4.0 International License (https://creativecommons.org/licenses/by-nc-sa/4.0/), which permits unrestricted NonCommercial use, distribution and reproduction in any medium, provided the original work is properly cited. 
acetophenone in the presence of opportunity base (aldol conditions) ${ }^{5}$. Recently, the heterocyclic compound of different ring with different heteroatoms are important for biologically active and industrial chemical products ${ }^{6}$. Pyrazoline (Py) and Pyrimidine (Pyr) derivatives are among the most prominent heterocyclic compounds, which have an important role in pharmaceutical and agrochemical industries as well as bioactive products because of their significant and wide spectrum of biological activities ${ }^{7}$.

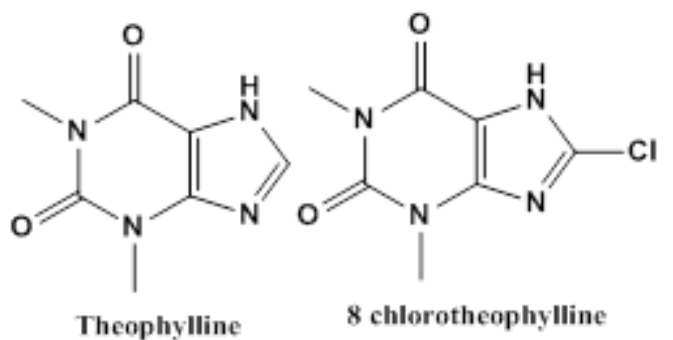

EXPERIMENTAL

All materials were of highest purity and supplied by Merck \& Co. Sigma-Aldrich and Flukacompany. Melting points were measured on a Buchi melting point apparatus B-545 (Buchi Labortechnik AG, Switzerland). Microanalytical data were obtained with a Vario, Elementar apparatus (Shimadzu, Japan). The IR spectra were recorded on Schimadzu, Fourier Transform Infra-red spectrophotometer (Model 270), using $\mathrm{KBr}$ discs. NMR spectra were recorded on $600 \mathrm{MHz}(1 \mathrm{H})$ and at $100 \mathrm{MHz}$ and $\left({ }^{13} \mathrm{C}\right)$ spectrometers (Bruker, Germany) with TMS as the internal standard and on ä scale in ppm. (TLC) was performed on silica gel for (TLC) and spots were visualized by lodine vapors. The reagents used were of analytical grade while the solvents were purified before use.

Synthesis of 8-(4-acetylphenylamino) -1,3dimethyl-1H-purine-2,6 (3H,7H) -dione (S1). ${ }^{8}$

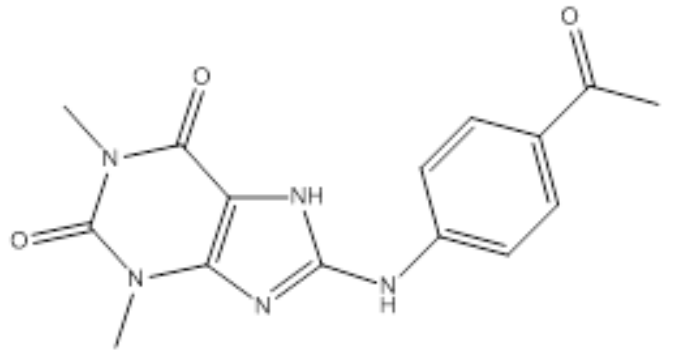

8-CTh $(0.21 \mathrm{~g} / 0.001 \mathrm{~mol})$ it was dissolve with 4-amino acetophenone $(0.135 \mathrm{~g} / 0.001 \mathrm{~mol})$ in $15 \mathrm{ml}$ of THF and add $1 \mathrm{ml}$ of triethyl amine and then reflux reaction for $24 \mathrm{~h}$ and monitored reaction by TLC using the solution (ethyl acetate: $\mathrm{n}$-Hexane) $(4: 2)$ the reaction is neutralized by glacial acetic acid and the precipitate obtained was filtered, washed and recrystallized from ethanol.Yield (79\%) as light yellow solid. m.p. $=176-179^{\circ} \mathrm{C} . R f=0.6, \mathrm{IR}$ $\left(\mathrm{KBr}, \mathrm{cm}^{-1}\right)$ : N-H (3361), C-H $\mathrm{H}_{\text {arom. }}$ (3130), C-H $\mathrm{H}_{\text {ali. }}(2983$, 2883), $\mathrm{C}=\mathrm{O}$ (1716), $\mathrm{C}=\mathrm{O}_{\text {ketone }}(1700) \mathrm{C}=\mathrm{C}_{\text {arom }}$ (1696), $\mathrm{C}=\mathrm{C}$ (1635) C-N (1373), $\mathrm{C}=\mathrm{N}(1519) .{ }^{1} \mathrm{H}-\mathrm{NMR}(600$ $\left.\mathrm{MHz}, \mathrm{DMSO}-d_{6}\right) \delta=3.18\left(\mathrm{~s}, 3 \mathrm{H}, \mathrm{N}_{1}-\mathrm{CH}_{3}\right), 3.53$ (s, $\left.3 \mathrm{H}, \mathrm{N}_{2}-\mathrm{CH}_{3}\right), 9.72\left(\mathrm{~s}, 1 \mathrm{H} \mathrm{N} \mathrm{N}_{10} \mathrm{H}\right), 14.54$ (s,br, $1 \mathrm{H}$, $\mathrm{N}_{9} \mathrm{H}$ ), 2.33 (s, 3H, COMe), 7.57-7.60 (d, 2H, C2' +6 ' $J=8.3 \mathrm{~Hz}), 7.89-7.91\left(\mathrm{~d}, 2 \mathrm{H}, \mathrm{C}^{\prime}+5^{\prime} \mathrm{J}=8.3 \mathrm{~Hz}\right){ }^{13} \mathrm{C}-$ NMR (100 MHz, DMSO $\left.d_{6}\right): \delta=27.3\left(\mathrm{~N}_{1}-\mathrm{CH}_{3}\right), 29.8$ $\left(\mathrm{N}_{3}-\mathrm{CH}_{3}\right), 24.6(\mathrm{Me}), 113.9\left(\mathrm{C}^{\prime}+6^{\prime}\right), 115.0(\mathrm{C} 5)$, $127.3\left(\mathrm{C}^{\prime}\right), 131\left(\mathrm{C}^{\prime}+5^{\prime}\right), 143.8\left(\mathrm{C}^{\prime}\right), 149.4(\mathrm{C} 4), 151.4$ (C=O2), 156.5 (C=O 6) , $167.3(\mathrm{C} 8), 196.5(\mathrm{C}=\mathrm{O})$.

\section{General procedure for synthesis chalcone (S2-} S4). ${ }^{9,10}$

$(3.13 \mathrm{~g} / 0.01 \mathrm{~mol})$ from S1 with different aromatic aldehydes $(0.01 \mathrm{~mol})$. The mixture dissolved in $10 \mathrm{ml}$ of alcohol. Sodium hydroxide solution $3 \mathrm{ml}(10 \%)$ was added slowly and the mixture stirred for $2 \mathrm{~h}$ until the entire mixture becomes very cloud then the mixture was poured slowly into $20 \mathrm{ml}$ of water with constant stirring and kept in refrigerator for 24 hours. The reaction was monitored by TLC (ethyl acetate: n-Hexane) (4:2). the reaction is neutralized by glacial acetic acid and, the precipitate obtained was filtered, washed and recrystallized from ethanol

8-(4-((E)-3-(4-hydroxy-3-methoxy phenyl) acryloyl) phenylamino)-1,3-dimethyl-1H-purine 2,6 (3H,7H)-dione (S2).

$(3.13 \mathrm{~g} / 0.01 \mathrm{~mol})$ of $\mathrm{S} 1$ with Vanillin $(1.52 \mathrm{~g} / 0.01 \mathrm{~mol})$ Yield $(74 \%)$ as Brown solid. m.p = $185-188^{\circ} \mathrm{C} . R f=0.51, \mathrm{FTIR}\left(\mathrm{KBr}, v, \mathrm{~cm}^{1}\right): 3336(\mathrm{~N}-$ $\mathrm{H}), 3113,2999,2885(\mathrm{C}-\mathrm{H})_{\text {ali }}, 1516(\mathrm{C}=\mathrm{C})_{\text {cha }}, 1668$ $(\mathrm{C}=\mathrm{O})_{\mathrm{cha}}, 3437(\mathrm{O}-\mathrm{H}), 1593,1471(\mathrm{C}=\mathrm{C})_{\mathrm{ar}} .1267(\mathrm{C}-$ $\mathrm{O}-\mathrm{C}), 3224\left(\mathrm{~N}_{10}-\mathrm{H}\right)$ cha. ${ }^{1} \mathrm{H}-\mathrm{NMR}(600 \mathrm{MHz}$, DMSO$\left.d_{6}\right) \delta=3.21\left(\mathrm{~s}, 3 \mathrm{H}, \mathrm{N}_{1}-\mathrm{CH}_{3}\right), 3.60\left(\mathrm{~s}, 3 \mathrm{H}, \mathrm{N}_{3}-\mathrm{CH}_{3}\right)$, 3.80 (s, 3H, OMe), 7.64, 7.66 (d, 1H, Hb J=15.9 Hz), $8.0,8.03(\mathrm{~d}, 1 \mathrm{H}, \mathrm{Ha} J=15.9 \mathrm{~Hz}), 9.84(\mathrm{~s}, 1 \mathrm{H}, \mathrm{OH})$, 12.46 (s. br, $1 \mathrm{H}, \mathrm{N}_{9} \mathrm{H}$ ), 9.64 (s. br, $1 \mathrm{H}, \mathrm{N}_{10} \mathrm{H}$ ), 6.54$7.44\left(\mathrm{~m}, 7 \mathrm{H}_{\text {arom }}\right) .{ }^{13} \mathrm{C}-\mathrm{NMR}\left(100 \mathrm{MHz}\right.$, DMSO $\left.d_{6}\right)$ $\delta=27.6\left(\mathrm{~N}_{1}-\mathrm{CH}_{3}\right), 29.7\left(\mathrm{~N}_{3}-\mathrm{CH}_{3}\right), 55.4(\mathrm{OMe}), 108.8$ 
(C5), 112.6 (C2"), $114.9\left(\mathrm{C2}^{\prime}+\mathrm{C6}^{\prime}+\mathrm{C}^{\prime \prime}\right), 121.5$ (Ca +C6" ), 123.3 (C1"), 124.8 (C4'), $130.4\left(\mathrm{C3}^{\prime}+\mathrm{C} 5^{\prime}\right)$, $144.2\left(\mathrm{C}^{\prime}+\mathrm{Cb}\right), 147.1(\mathrm{C} 4), 147.3\left(\mathrm{C} 4^{\prime \prime} \mathrm{C}-\mathrm{OH}\right)$, 150.8 (C3'(C-OMe) ,151.0 ( $\mathrm{C}=\mathrm{O} 2), 153.8(\mathrm{C}=\mathrm{O} 6)$, $167.1(\mathrm{C} 8), 194.8(\mathrm{C}=\mathrm{O})$ cha. Anal. calc. for $\mathrm{C}_{23} \mathrm{H}_{21} \mathrm{~N}_{5} \mathrm{O}_{5}$ (447.45): C 61.74, H 4.73, N 15.65. Found: 61.51, H 4.65, N 15.42 .

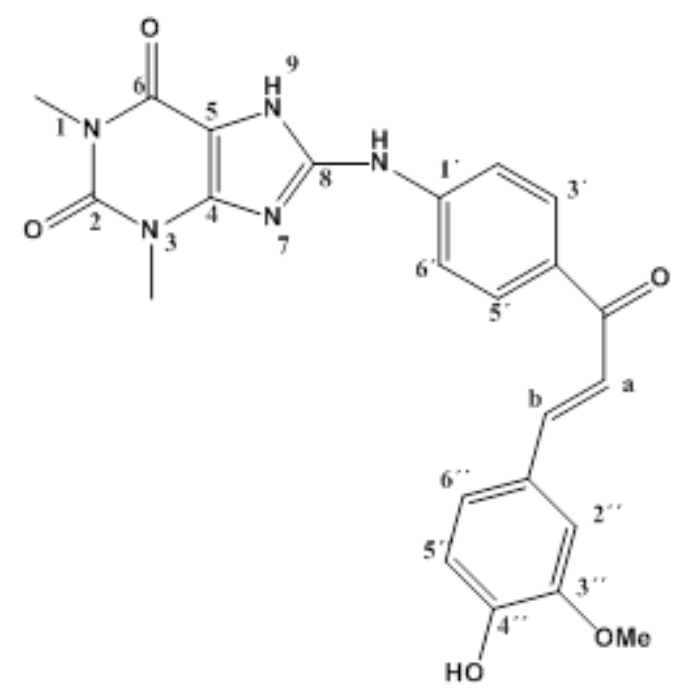

8-(4-((E)-3-(4-chlorophenyl) acryloyl) phenylamino)1,3-dimethyl - $1 \mathrm{H}$-purine-2,6 (3H,7H)-dione (S3).

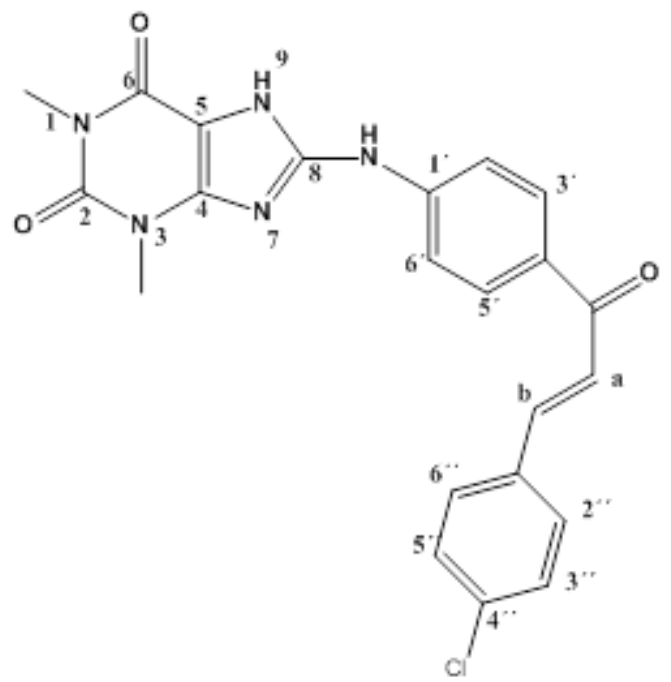

(3.13 $\mathrm{g} / 0.01 \mathrm{~mol})$ from S1 with $\mathrm{p}$-chloro benzaldehyde $(1.4 \mathrm{~g} / 0.01 \mathrm{~mol})$ Yield $(82 \%)$ as Yellow solid. m.p $=178-181^{\circ} \mathrm{C}$. $R f=0.55$ FTIR $\left(\mathrm{KBr}, v, \mathrm{~cm}^{1}\right)$ : $3460(\mathrm{~N}-\mathrm{H}), 2995,2875(\mathrm{C}-\mathrm{H})_{\text {ali, }}, 1589$ $(\mathrm{C}=\mathrm{C})_{\text {cha }}, 1665(\mathrm{C}=\mathrm{O})_{\text {cha }}, 1055(\mathrm{C}-\mathrm{O}), 1016(\mathrm{C}-\mathrm{Cl})_{\text {cha }}$, $3219\left(\mathrm{~N}_{10}-\mathrm{H}\right) .{ }^{1} \mathrm{H}$ NMR $\left(600 \mathrm{MHz}\right.$, DMSO- $\left.d_{6}\right) \mathrm{d}=3.17$ (s, $3 \mathrm{H}, \mathrm{N}_{1}-\mathrm{CH}_{3}$ ), 3.33 (s, 3H, $\mathrm{N}_{3}-\mathrm{CH}_{3}$ ), 7.33-7.68 $(\mathrm{m}, 8 \mathrm{H}$. arom $), 7.72,7.76(\mathrm{~d}, 1 \mathrm{H}, \mathrm{Hb} J=15.6 \mathrm{~Hz})$,
7.99,8.03 (d,1H, Ha J=15.6 Hz), 8.69 (s, br, 1H, $\mathrm{NH}), 9.99\left(\mathrm{~s}, 1 \mathrm{H}, \mathrm{N}_{9} \mathrm{H}\right){ }^{13} \mathrm{C}-\mathrm{NMR}(100 \mathrm{MHz}$, DMSO $\left.d_{6}\right) d=27.3\left(\mathrm{~N}_{1}-\mathrm{CH}_{3}\right), 29.8\left(\mathrm{~N}_{3}-\mathrm{CH}_{3}\right), 115.0(\mathrm{C} 5)$, $112.7\left(\mathrm{C}^{\prime}+\mathrm{C}^{\prime}\right), 121.3(\mathrm{Ca}), 127.3\left(\mathrm{C} 4^{\prime}\right), 129.3$ (C3"+C5"), 130.6 (C2"+C6"), $131.1\left(\mathrm{C3}^{\prime}+\mathrm{C} 5^{\prime}\right), 134.6$ (C4" CCl+C1"), 142.7 (C1'), 144.6 (Cb), 149.4 (C4), 151.4 ( $\mathrm{C}=\mathrm{O} 2$ ), 156.5 ( $\mathrm{C}=\mathrm{O}$ 6), 166.9 (C8), 192.6 $\left(\mathrm{C}=\mathrm{O}_{\text {cha }}\right)$. Anal. calc. for $\mathrm{C}_{22} \mathrm{H}_{18} \mathrm{ClN}_{5} \mathrm{O}_{3}$ (435.87): $\mathrm{C}$ 60.62, H 4.16, N 16.07. Found: C 60.40, H 4.04, N 15.79 .

8-(4-((E)-3-(4-(dimethylamino) phenyl) acryloyl) phenylamino)-1,3-dimethyl-1H-purine 2,6(3H,7H) dione (S4)<smiles>CN(C)c1ccc(/C=C/C(=O)c2ccc(Nc3nc4c([nH]3)c(=O)n(C)c(=O)n4C)cc2)cc1</smiles>

(3.13 g / $0.01 \mathrm{~mol})$ from S1 with $\mathrm{p}$-dimethyl amino benzaldehyde $(1.49 \mathrm{~g} / 0.01 \mathrm{~mol})$ Yield $(78 \%)$ as Brown solid. m.p $=189-191^{\circ} \mathrm{C} . R f=0.42$ FTIR $\left(\mathrm{KBr}, \mathrm{v}, \mathrm{cm}^{1}\right): 3336(\mathrm{~N}-\mathrm{H}), 3132,2939,2858(\mathrm{C}-\mathrm{H})$ ali, $3000(\mathrm{C}-\mathrm{H}) 1540(\mathrm{C}=\mathrm{C})_{\text {cha }}, 1658(\mathrm{C}=\mathrm{O})_{\text {cha }}, 1604$ $(\mathrm{C}=\mathrm{C})_{\mathrm{ar}}{ }^{1} \mathrm{H}-\mathrm{NMR}:\left(600 \mathrm{MHz}, \mathrm{DMSO}-d_{6}\right) \delta=3.38$ (s, $3 \mathrm{H}, \mathrm{N}_{1}-\mathrm{CH}_{3}$ ), 3.21 (s, 3H, N $\mathrm{CH}_{3}$ ), $3.06(\mathrm{~s}, 3 \mathrm{H}$, NMe2), 7.50,7.51 (d,1H, Hb), $8.06,8.08(\mathrm{~d}, 1 \mathrm{H}, \mathrm{Ha})$, 6.54-6.56 (d,2H, C3"+C5"), 7.60-7.62 (d, 2H, $\left.\mathrm{C3}^{\prime}+\mathrm{C5}^{\prime}\right), 7.64-7.66\left(\mathrm{~d}, 2 \mathrm{H}, \mathrm{C}^{\prime}{ }^{\prime}+6^{\prime}\right), 7.72-7.74$ (C2"+C6"), $9.84\left(\mathrm{~s}, 1 \mathrm{H}, \mathrm{N}_{9} \mathrm{H}\right), 12.46(\mathrm{~s}, \mathrm{br}, 1 \mathrm{H}, \mathrm{NH})$. ${ }^{13} \mathrm{C}-\mathrm{NMR}\left(100 \mathrm{MHz}\right.$, DMSO- $\left.d_{6}\right) \delta=27.3\left(\mathrm{~N}_{1}-\mathrm{CH}_{3}\right)$, $29.8\left(\mathrm{~N}_{3}-\mathrm{CH}_{3}\right), 43.5\left(\mathrm{NMe}_{2}\right), 112.5$ (C5), 111.9 $\left(\mathrm{C} 2^{\prime}+\mathrm{C}^{\prime}\right), 122.9$ (Ca) ,129.4 (C4'), 111.5 (C3"+C5"), $129.7\left(\mathrm{C}^{\prime \prime}+\mathrm{C} 6 "\right), 130.1\left(\mathrm{C3}^{\prime}+\mathrm{C} 5^{\prime}\right), 151.4$ (C4"), 123.3 (C1") 141.4 (C1'), $143.9(\mathrm{Cb}), 148.1$ (C4), $150.1(\mathrm{C}=\mathrm{O} 2), 156.2(\mathrm{C}=\mathrm{O}$ 6), 167.2 (C8), $191.5(\mathrm{C}=\mathrm{O})$ cha: 
General procedure for synthesis Pyrimidine compounds derivatives (S5-S7). ${ }^{11}$

$(0.001 \mathrm{~mol})$ of prepared chalcone was dissolved in ethanolic sodium hydroxide $(10 \mathrm{ml})$ and $(0.001 \mathrm{~mol})$ was add slowly from thiourea, were stirred about 3-4 $\mathrm{h}$ with a magnetic stirrer. This was then poured into $5 \mathrm{ml}$ of cold water with continuous stirring for an $\mathrm{h}$ and then kept in refrigerator for $24 \mathrm{~h}$, the reaction was monitored by TLC (ethyl acetate: n-Hexane) (4:2). The precipitate obtained was filtered, washed and recrystallized with ethanol.

8-(4-(6-(4-hydroxy-3-methoxy phenyl)-3,6dihydro-2-mercapto pyrimidin-4-yl) phenyl amino)-1,3-dimethyl-1H-purine-2,6(3H,7H)-dione (S5)

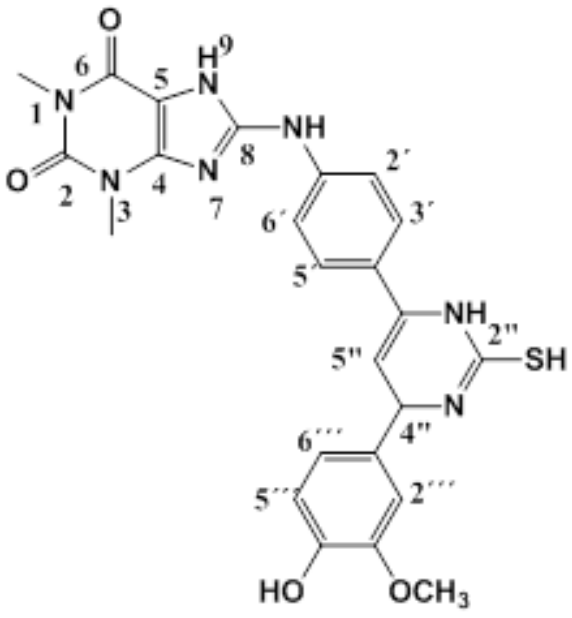

$(0.447 \mathrm{~g} / 0.001 \mathrm{~mol})$ from S2 with thiourea (0.076 g / $0.001 \mathrm{~mol})$ Yield (72\%) as light Brown solid. m.p $=195-199^{\circ} \mathrm{C} . R f=0.35$, FTIR $(\mathrm{KBr}, v$, $\left.\mathrm{cm}^{1}\right), 2644(\mathrm{~S}-\mathrm{H}), 3394(\mathrm{O}-\mathrm{H}), 1593(\mathrm{C}=\mathrm{C})_{\mathrm{ar}} 2993$ $(\mathrm{C}-\mathrm{H})_{\text {ar. }} 1099(\mathrm{C}-\mathrm{O}-\mathrm{C}), 1523(\mathrm{C}=\mathrm{N})_{\text {pyr. }} 1648(\mathrm{C}=\mathrm{C})_{\text {pyr }}$. ${ }^{1} \mathrm{H}-\mathrm{NMR}\left(600 \mathrm{MHz}, \mathrm{DMSO}-d_{6}\right) \delta=1.36(\mathrm{~s}, 1 \mathrm{H}, \mathrm{SH})$ $3.34\left(\mathrm{~s}, 3 \mathrm{H}, \mathrm{N}_{1}-\mathrm{CH}_{3}\right), 3.50\left(\mathrm{~s}, 3 \mathrm{H}, \mathrm{N}_{3}-\mathrm{CH}_{3}\right), 3.93$ (s,3H. OMe), 4.78 (s.br, $\left.1 \mathrm{H}, \mathrm{C}_{5^{\prime \prime}}\right), 5.20$ (s.br, $1 \mathrm{H}, \mathrm{C}_{2 "}$ ) 6.68 (s, br.,1H, C64), 6.88 (s,1H, br, $\left.{ }_{\mathrm{c} 44}\right), 6.96$ (s, $\left.\mathrm{br}, 1 \mathrm{H}, \mathrm{C}_{24}\right), 7.56\left(\mathrm{~s}, 1 \mathrm{H}, \mathrm{C}_{3^{\prime}+5}\right), 7.78\left(\mathrm{~s}, \mathrm{br}, 1 \mathrm{H}, \mathrm{c}^{\prime}+6^{\prime}\right)$ 8.18 (s , 1 H NHpyr), 9.07 (s, br, $\left.1 \mathrm{H}, \mathrm{N}_{9} \mathrm{H}\right), 9.66(\mathrm{~s}, 1 \mathrm{H}$, $\mathrm{OH}), 11.38\left(\mathrm{~s}, 1 \mathrm{H}, \mathrm{N}_{10} \mathrm{H}\right) \cdot{ }^{13} \mathrm{C}-\mathrm{NMR}(100 \mathrm{MHz}$, DMSO $\left.d_{6}\right): \mathrm{d}=27.6\left(\mathrm{~N}_{1}-\mathrm{CH}_{3}\right), 29.7\left(\mathrm{~N}_{3}-\mathrm{CH}_{3}\right), 55.4(\mathrm{OMe})$, 62.7 (C2"), 103.4 (C5"), 108.1 (C5), 112.3 (C24), 114.9 (C54), $121.5\left(\mathrm{C}^{\prime}+\mathrm{C}^{\prime}\right), 123.3$ (C64), 130.4 $\left(\mathrm{C3}^{\prime}+\mathrm{C}^{\prime}\right), 125.9$ (C4'), 133.4 (C14), 136.5 (C1'), 142.3 (C6"), 148.8 (C34+C44), 149.7 (C4), 150.8 (C=O 2) 153.6 (C=O 6), 164.6 (C4"), 167.1 (C8). Anal. calc. for $\mathrm{C}_{24} \mathrm{H}_{25} \mathrm{~N}_{7} \mathrm{O}_{4} \mathrm{~S}$ (507.57): 56.79, $\mathrm{H} 4.96$, N 19.32. Found: C 56.56, H 4.82, N 19.09 . 8-(4-(6-(4-chlorophenyl)-3,6-dihydro-2mercaptopyrimidin-4-yl) phenylamino)-1,3dimethyl-1H-purine-2,6(3H,7H)-dione (S6)

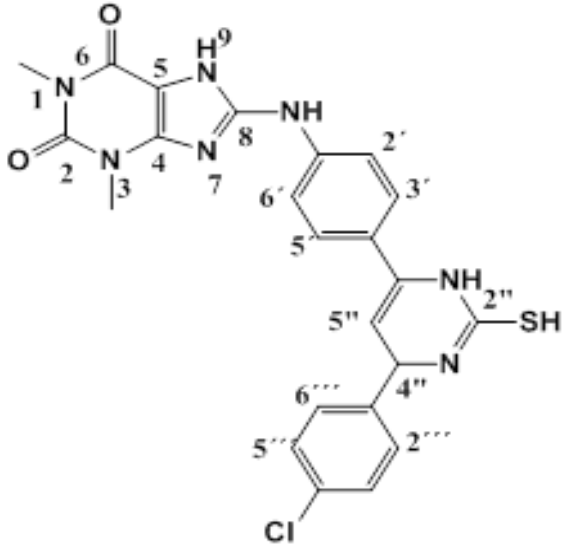

$(0.435 \mathrm{~g} / 0.001 \mathrm{~mol})$ from S3 with thiourea $(0.076 \mathrm{~g} / 0.001 \mathrm{~mol})$ Yield $(69 \%)$ as Brown solid. $\mathrm{m} . \mathrm{p}=190-192{ }^{\circ} \mathrm{C} . R f=0.29$, $\mathrm{FT}$ IR $\left(\mathrm{KBr}, v, \mathrm{~cm}^{1}\right)$ : $2653(\mathrm{~S}-\mathrm{H}), 1604(\mathrm{C}=\mathrm{C})$ ar $1552(\mathrm{C}=\mathrm{N}) 3084(\mathrm{C}-\mathrm{H})$ ar. $1523(\mathrm{C}=\mathrm{N})$ pyr. , $1652(\mathrm{C}=\mathrm{C})$ pyr. ${ }^{1} \mathrm{H}-\mathrm{NMR}(600$ $\left.\mathrm{MHz}, \mathrm{DMSO}-d_{6}\right) \delta=1.41(\mathrm{~s}, 1 \mathrm{H}, \mathrm{SH}), 3.20\left(\mathrm{~s}, 3 \mathrm{H}, \mathrm{N}_{1}-\right.$ $\left.\mathrm{CH}_{3}\right), 3.36\left(\mathrm{~s}, 3 \mathrm{H}, \mathrm{N}_{3}-\mathrm{CH}_{3}\right), 4.65\left(\mathrm{~s}, 1 \mathrm{H}, \mathrm{C}_{5^{\prime \prime}}\right), 5.23(\mathrm{~s}$, $\left.1 \mathrm{H}, \mathrm{C}_{2^{n}}\right), 7.09-7.13(\mathrm{~d}, 1 \mathrm{H}, \mathrm{C} 24+64 \mathrm{~J}=7.9 \mathrm{~Hz}), 7.21$ $7.24\left(\mathrm{~d}, 1 \mathrm{H}, \mathrm{C3}^{\prime}+5^{\prime} \mathrm{J}=8.2 \mathrm{~Hz}\right), 7.41-7.44(\mathrm{~d}, 1 \mathrm{H}$, C34+54 J=7.9 Hz), 7.57-7.60 (d, 1H, C2' $+6{ }^{\prime} J=8.2$ $\mathrm{Hz}$ ), 8.71 (s ,1H NH), 9.34 (s,br, $1 \mathrm{H}, \mathrm{N}_{9} \mathrm{H}$ ), 10.38 (s, $\left.1 \mathrm{H}, \mathrm{N}_{10} \mathrm{H}\right) \cdot{ }^{13} \mathrm{C}-\mathrm{N} M R\left(100 \mathrm{MHz}\right.$, DMSO $\left.d_{6}\right) \mathrm{d}=27.5$ $\left(\mathrm{N}_{1}-\mathrm{CH}_{3}\right), 29.7\left(\mathrm{~N}_{3}-\mathrm{CH}_{3}\right), 65.4(\mathrm{C} 2 "), 102.9(\mathrm{C} 5 ")$, 110.6 (C5), $120.8\left(\mathrm{C}^{\prime}+\mathrm{C}^{\prime}\right), 123.9$ (C34+C54), 128.7 (C3'+ C5'), 129.4 (C24+C64), 130.1 (C4'), 131.1 (C14+C44), 135.6 (C1'), 142.9 (C6"), 146.6 (C4), 151.4 (C=O 2), 153.8 (C=O 6), 159.4 (C4"), 166.8 (C8)

8-(4-(6-(4-(dimethyl amino) phenyl)-3,6-dihydro2-mercaptopyrimidin-4yl)phenylamino)-1,3dimethyl-1H-purine-2,6(3H,7H)-dione (S7)

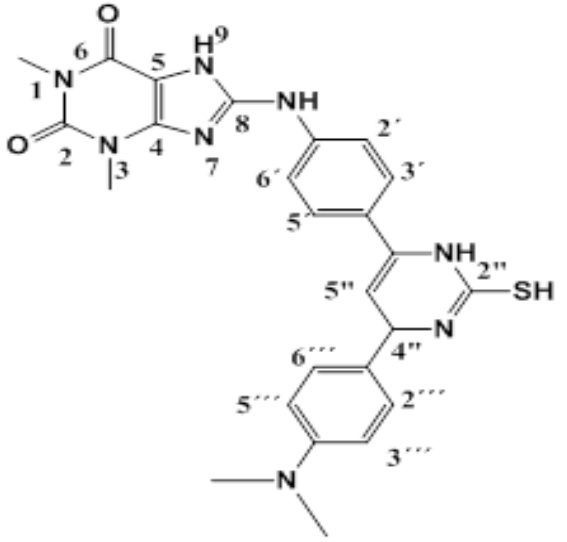


$(0.444 \mathrm{~g} / 0.001 \mathrm{~mol})$ from S4 with thiourea $(0.076 \mathrm{~g} / 0.001 \mathrm{~mol})$ Yield $(78 \%)$ as Brown solid. $\mathrm{m} . \mathrm{p}=198-200^{\circ} \mathrm{C} . R f=0.25, \mathrm{FTIR}\left(\mathrm{KBr}, \mathrm{v}, \mathrm{cm}^{1}\right): 2646$ (S-H) $1604(\mathrm{C}=\mathrm{C})$ ar $3050(\mathrm{C}-\mathrm{H})$ ar. $1500(\mathrm{C}=\mathrm{N})$ pyr. , $1649(\mathrm{C}=\mathrm{C})$ pyr. ${ }^{1} \mathrm{H}-\mathrm{NMR}\left(600 \mathrm{MHz}\right.$, DMSO- $\left.d_{6}\right) \delta=$ 1.37 (s, 1H, SH), 3.16 (s, 6H, NMe2) 3.20 (s, 3H, $\left.\mathrm{N} 1-\mathrm{CH}_{3}\right), 3.32\left(\mathrm{~s}, 3 \mathrm{H}, \mathrm{N} 3-\mathrm{CH}_{3}\right), 4.68(\mathrm{~s}, 1 \mathrm{H}, \mathrm{C} 5 ")$, 5.25 (s, 1H, C2") 6.87-6.89 (d, 2H, C24+C64 J=7.8 $\mathrm{Hz}), 7.09-7.13\left(\mathrm{~d}, 2 \mathrm{H}, \mathrm{C3}^{\prime}+5^{\prime} \mathrm{J}=8.2 \mathrm{~Hz}\right), 7.22-7.24$ (d, $2 \mathrm{H}, \mathrm{C} 34+54 \mathrm{~J}=7.8 \mathrm{~Hz}), 7.34-7.36$ (d, 2H, C2' $+6{ }^{\prime}$ $\mathrm{J}=8.2 \mathrm{~Hz}), 9.57(\mathrm{~s}, 1 \mathrm{H}, \mathrm{NH}), 9.76\left(\mathrm{~s}, 1 \mathrm{H}, \mathrm{N}_{9} \mathrm{H}\right), 12.41$ $(\mathrm{s}, 1 \mathrm{H}, \mathrm{NH}) \cdot{ }^{13} \mathrm{C}-\mathrm{NMR}\left(100 \mathrm{MHz}\right.$, DMSO $\left.d_{6}\right): \delta=27.7$ (N1-CH3), 29.8 (N3-CH3), 43.6 (NMe2) , 65.8 (C2"), 103.6 (C5"), 111.1 (C34+C54), 112.6 (C5), 120.6 $\left(\mathrm{C}^{\prime}+\mathrm{C6}^{\prime}\right), 124.1$ (C14), $128.7\left(\mathrm{C3}^{\prime}+\mathrm{C}^{\prime}\right), 129.4$ (C24+C64), 130.8 (C4'), 135.6 (C1'), 142.9 (C6"), 144.4 (C4), 146.4 (C44), $152.6(\mathrm{C}=\mathrm{O} 2), 154.8$ ( $\mathrm{C}=\mathrm{O}$ 6), 159.6 (C4"), 167.5 (C8).

\section{General procedure for synthesis pyrazoline compounds derivatives (S8-S10) ${ }^{12}$}

A mixture a prepared chalcone $(0.001$ $\mathrm{mol})$, and $(0.001 \mathrm{~mol})$ and hydrazine hydrate $(99 \%)$ $(0.001 \mathrm{~mol})$ in $(25 \mathrm{ml})$ ethanol was refluxed with stirring about $5 \mathrm{hrs}$, the reaction was monitored by TLC (ethyl acetate: n-Hexane) (4:2). The precipitate obtained was filtered, washed and recrystallized with ethanol.

8-(4-(4,5-dihydro-5-(4-hydroxy-3-methoxyphenyl)1H-pyrazoline-3-yl) phenylamino)-1,3-dimethyl1H-purine-2,6(3H,7H)-dione. (S8)

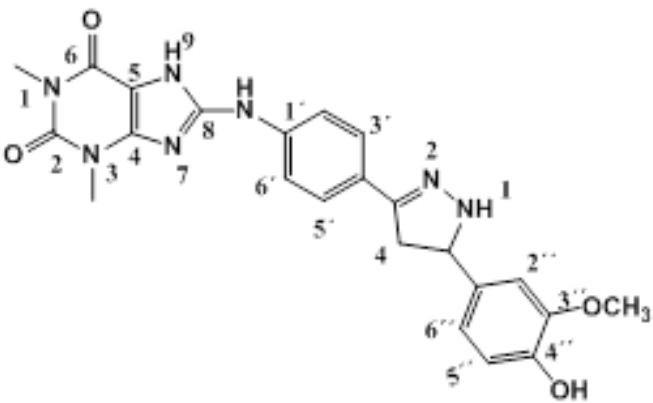

$(0.447 \mathrm{~g} / 0.001 \mathrm{~mol})$ from S2 with hydrazine $(0.032 \mathrm{~g} / 0.001 \mathrm{~mol})$ Yield $(80 \%)$ as brown solid. m.p $=191-193{ }^{\circ} \mathrm{C} . R f=0.38 \mathrm{FTIR}(\mathrm{KBr}, v$, $\left.\mathrm{cm}^{1}\right)$ : 2960, 2926, $2879(\mathrm{C}-\mathrm{H})_{\text {ali }}, 3363,3219(\mathrm{~N}-\mathrm{H})$ Py, $3039,1595(\mathrm{C}=\mathrm{C})_{\text {ar }}, 3069(\mathrm{C}-\mathrm{H})_{\text {ar.Py }}, 3462(\mathrm{~N}-$ $\mathrm{H}), 3564(\mathrm{O}-\mathrm{H}) .{ }^{1} \mathrm{H}$ NMR $\left(600 \mathrm{MHz}\right.$, DMSO- $\left.d_{6}\right)=$ 3.22 (s, 3H, $\mathrm{N}_{1}-\mathrm{CH}_{3}$ ), 3.37 (s, 3H, $\left.\mathrm{N}_{3}-\mathrm{CH}_{3}\right) 3.63$ (s, $3 \mathrm{H}, \mathrm{OMe}), 3.78$ (s, $1 \mathrm{H}, \mathrm{C} 5 \mathrm{py}), 3.82\left(\mathrm{~s}, 1 \mathrm{H}, \mathrm{C}_{4} \mathrm{Py}\right)$,
6.77-7.83 (d, 8H. arom), 9.23 (s , $\left.1 \mathrm{H} \mathrm{NH}_{\text {py. }}\right), 10.29$ $\left(\mathrm{s}, 1 \mathrm{H}, \mathrm{N}_{9} \mathrm{H}\right), 13.38(\mathrm{~s} 1 \mathrm{H}, \mathrm{NH}), 9.50(\mathrm{~s}, 1 \mathrm{H}, \mathrm{OH}),{ }^{13} \mathrm{C}-$ NMR (100 MHz, DMSO $\left.d_{6}\right): \delta=27.6\left(\mathrm{~N}_{1}-\mathrm{CH}_{3}\right), 29.7$ $\left(\mathrm{N}_{3}-\mathrm{CH}_{3}\right), 42.4\left(\mathrm{C}_{4 \mathrm{Py}}\right), 58.8(\mathrm{OMe}), 49.7\left(\mathrm{C}_{5 \mathrm{py}}\right), 108.8$ (C5), 112.6 (C2") ,114.9 (C2'+C6'+ C5"), 121.5 (C6") ,123.3 C4') ,130.4 (C3'+C5'), 137.5 (C1"), 142.6 (C1'), 147.5 (C4" C-OH ) ,147.7 (C4), 149.7 (C3"), $150.8\left(\mathrm{C}_{\text {зру }}\right), 150.0\left(\mathrm{C}=\mathrm{O} 2+\mathrm{C}^{\prime}(\mathrm{C}-\mathrm{OMe}), 151.0\right.$ $(\mathrm{C}=\mathrm{O}$ 6) ,168.2(C8).

8-(4-(5-(4-chlorophenyl)-4,5-dihydro-1H-pyrazoline3-yl) phenylamino)-1,3-dimethyl-1H-purine2,6(3H,7H)-dione (S9)

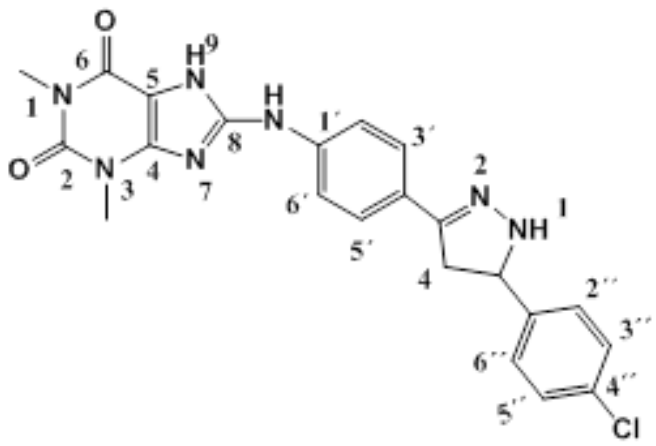

$(0.435 \mathrm{~g} / 0.001 \mathrm{~mol})$ from S3 with hydrazine $(0.032 \mathrm{~g} / 0.001 \mathrm{~mol})$ Yield $(77 \%)$ as Yellow solid. m.p $=189-193^{\circ} \mathrm{C} . R f=0.42 \mathrm{FTIR}(\mathrm{KBr}$, $\left.\mathrm{v}, \mathrm{cm}^{1}\right): 3130,2981,2926,2879(\mathrm{C}-\mathrm{H})$ ali, $3429(\mathrm{~N}-$ $\mathrm{H}), 3346,3227(\mathrm{~N}-\mathrm{H})$ py ,1597 (C=C) ar., $3462(\mathrm{~N}$ $\mathrm{H}), 3059(\mathrm{C}-\mathrm{H})$ ar. ${ }^{1} \mathrm{H}-\mathrm{NMR}\left(600 \mathrm{MHz}, \mathrm{DMSO}-d_{6}\right)$ $\delta=3.21(\mathrm{~s}, 3 \mathrm{H}, \mathrm{N} 1-\mathrm{CH} 3), 3.37\left(\mathrm{~s}, 3 \mathrm{H}, \mathrm{N} 3-\mathrm{CH}_{3}\right)$, 3.87 (d, 1H, C5), 7.43-7.85 (m, 8H. arom), 9.15 (s , 1 H $\left.\mathrm{NH}_{\text {py }}\right), 10.09\left(\mathrm{~s}, 1 \mathrm{H}, \mathrm{N}_{9} \mathrm{H}\right), 3.71\left(\mathrm{~s}, 1 \mathrm{H}, \mathrm{C}_{4} \mathrm{Py}\right) \cdot{ }^{13} \mathrm{C}-$ NMR (100 MHz, DMSO $\left.d_{6}\right): \delta=27.6\left(\mathrm{~N}_{1}-\mathrm{CH}_{3}\right), 29.7$ $\left(\mathrm{N}_{3}-\mathrm{CH}_{3}\right), 108.5(\mathrm{C} 5), 113.1\left(\mathrm{C}^{\prime}+\mathrm{C}^{\prime}\right), 123.4\left(\mathrm{C}^{\prime}\right)$ ,127.5 (C3'+C5' +C2" +C6"), $129.4\left(\mathrm{C}^{\prime \prime}+\mathrm{C} 5^{\prime \prime}\right)$, 141.4 (C1"), 134.0 (C4"), 136.9 (C1'), 147.2 (C4), $150.2(\mathrm{C} 3), 48.6\left(\mathrm{C}_{5 \mathrm{Py}}\right), 151.7\left(\mathrm{C}_{3 \mathrm{py}}\right), 42.3\left(\mathrm{C} 4_{\mathrm{Py}}\right)$, 150.8 ( $\mathrm{C}=\mathrm{O} 2), 153.7$ ( $\mathrm{C}=\mathrm{O}$ 6), 167.1 (C8)

8-(4-(5-(4-(dimethylamino) phenyl)-4,5-dihydro1H-pyrazoline-3-yl) phenylamino)-1,3-dimethyl1H-purine -2,6(3H,7H) dione. (S10)

$(0.444 \mathrm{~g} / 0.001 \mathrm{~mol}$,$) from S4 with$ hydrazine $(0.032 \mathrm{~g} / 0.001 \mathrm{~m})$ Yield $(76 \%)$ as brown solid .m.p. $=199-102{ }^{\circ} \mathrm{C} . R f=0.61$ FTIR $(\mathrm{KBr}, v$, $\left.\mathrm{cm}^{1}\right)$, ,3334,3223 (N-H) $)_{\mathrm{py}}, 1597(\mathrm{C}=\mathrm{C})_{\mathrm{ar}}, 1560(\mathrm{C}=\mathrm{N})$ $3039(\mathrm{C}-\mathrm{H})_{\text {ar.Py }}, 3334(\mathrm{~N}-\mathrm{H})_{\mathrm{Py}}, 2953,2922,2856$ $(\mathrm{C}-\mathrm{H}){ }_{\text {ali }}{ }^{1} \mathrm{H}-\mathrm{NMR}\left(600 \mathrm{MHz}, \mathrm{DMSO}-d_{6}\right) \delta=2.98$ (s, $\left.3 \mathrm{H}, \mathrm{N}(\mathrm{Me})_{2}\right), 3.20\left(\mathrm{~s}, 3 \mathrm{H}, \mathrm{N}_{1}-\mathrm{CH}_{3}\right), 3.36\left(\mathrm{~s}, 3 \mathrm{H}, \mathrm{N}_{3}-\right.$ 
$\left.\mathrm{CH}_{3 .}\right), 3.47\left(\mathrm{~s}, 1 \mathrm{H}, \mathrm{C} 4{ }_{\mathrm{Py}}\right), 3.82\left(\mathrm{~s}, 1 \mathrm{H}, \mathrm{C} 5{ }_{\mathrm{Py}}\right), 6.57-$ $6.59\left(\mathrm{~d}, 2 \mathrm{H}, \mathrm{C} 3^{\prime \prime}+5 " \mathrm{~J}=8.6 \mathrm{~Hz}\right), 6.74-6.77(\mathrm{~d}, 2 \mathrm{H}$, $\left.\mathrm{C2}^{\prime}+6^{\prime} \mathrm{J}=8.5 \mathrm{~Hz}\right), 7.32-7.34$ (d, 2H, C2" $+6 " \mathrm{H}=8.6$ $\mathrm{Hz}), 7.61-7.64$ (d,2H, C3'+5' J=8.5 Hz), , 8.36 (s , $\left.1 \mathrm{H} \mathrm{NH}_{\mathrm{py}}\right), 10.60\left(\mathrm{~s}, 1 \mathrm{H}, \mathrm{N}_{9} \mathrm{H}\right), 13.06(\mathrm{~s}, 1 \mathrm{H}, \mathrm{NH}) 5.98$ $\left(\mathrm{s}, 1 \mathrm{H}, \mathrm{C}_{4}\right.$ Py $) \cdot{ }^{13} \mathrm{C}-\mathrm{NMR}\left(100 \mathrm{MHz}\right.$, DMSO $\left.d_{6}\right)$ : $\delta=27.5\left(\mathrm{~N}_{1}-\mathrm{CH}_{3}\right), 29.7\left(\mathrm{~N}_{3}-\mathrm{CH}_{3}\right), 45.5(\mathrm{NMe})_{2}, 43.0$ (C4) ${ }_{\mathrm{py}}, 49.9$ (C5) $)_{\mathrm{py}}, 108.2$ (C5), 113.0 (C3"+C5"), $118.1\left(\mathrm{C}^{\prime}+\mathrm{C}^{\prime}\right), 125.7\left(\mathrm{C}^{\prime}\right), 127.5\left(\mathrm{C}^{\prime}{ }^{\prime}+\mathrm{C}^{\prime}\right)$ 127.8 (C2"+C6"), 133.5 (C1"), 138.7 (C1'), 147.3 (C4), 149.0 (C4"), 150.2 (C3) py 151.4 (C=O 2), 153.4 $\left(\mathrm{C}=\mathrm{O} 6\right.$ ), 166.8 (C8). Anal. calc. for $\mathrm{C}_{24} \mathrm{H}_{24} \mathrm{~N}_{8} \mathrm{O}_{2}$ (456.20): 63.15, H5.30, N 24.55. Found: C 62.92, H 5.21, N 24.31

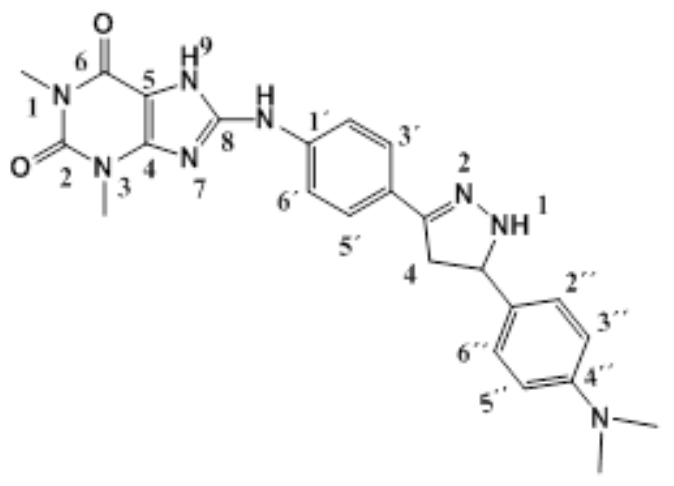

\section{Antimicrobial Evolution}

The newly synthesized compounds were selected for their antimicrobial activities against different bacteria and fungi. The microorganisms used were Staphylococcus aureus (Gram positive), Escherichia coli, (Gram negative) and Candida albicans by using the agar diffusion method ${ }^{13}$ to select the most potent compounds. $5 \mathrm{mg}$ of each compound was dissolved in dimethyl sulfoxide (DMSO, $1 \mathrm{~mL}$ ) then complete up to $10 \mathrm{~mL}$ with distilled water to give a concentration of $500 \sim \mathrm{g} / \mathrm{mL}$. The bacteria were maintained on Muller hentone agar media, the dishes incubated at $37{ }^{\circ} \mathrm{C}$ for $24 \mathrm{~h}$ for bacteria while $72 \mathrm{~h}$ for fungal. ${ }^{14,15}$ The efficiency of the tested compounds was compared to that of water and DMSO, zone of inhibition measured by ruler.

\section{RESULTS AND DICUSSION}

8-CTh was a starting material for the synthesis of new chalcone compound over a reaction with (P-amino acetophenone) scheme ${ }^{1 .}$ The appearance of stretching band of secondary aromatic amine $(\mathrm{NH})$ at (3361) $\mathrm{cm}^{-1}$ and disappearance of $\mathrm{C}-\mathrm{Cl}$ of $8-\mathrm{CTh}$ at $(760) \mathrm{cm}^{-1}$, and showed- ${ }^{1} \mathrm{H}-\mathrm{NMR}$ spectrum for $\mathrm{N}-\mathrm{H} 14.54$ (s, br, $1 \mathrm{H}$, $\mathrm{NH}$ ), are attributed to the formation amine compound. ${ }^{13} \mathrm{C}-N M R$ spectrum for $24.6(\mathrm{Me}), 113.9$ $\left(\mathrm{C}^{\prime}+6^{\prime}\right), 127.3\left(\mathrm{C}^{\prime}\right), 131\left(\mathrm{C}^{\prime}+5^{\prime}\right), 143.8\left(\mathrm{C} 1^{\prime}\right)^{16}$. The reaction of $\mathrm{S} 1$ compond with different aromatic aldehydes in the presence $\mathrm{NaOH}$ to formation chalcone in scheme 2 showed appearance of the band 1516, 1589 and 1525 to $(\mathrm{C}=\mathrm{C})$ cha for S2, S3 and $S 4$ respectively, in other hand 1668,1665 and 1658 to $(\mathrm{C}=\mathrm{O})$ cha. for $\mathrm{S} 2, \mathrm{~S} 3$ and S4 respectively. ${ }^{1} \mathrm{H}-\mathrm{NMR}$ showed the deplete of $\mathrm{CH}=\mathrm{CH}$ proton at about 7.64, 7.66 for $\mathrm{Hb}, 8.0,8.03$ for $\mathrm{Ha}$ to $\mathrm{S} 2$ and 7.72, 7.76 Hb, 7.99, 8.03 $\mathrm{Ha}$ to S3 and 7.50,7.51 for $\mathrm{Hb}, 8.06,8.08$ for $\mathrm{Ha}$ to $\mathrm{S} 4 .{ }^{13} \mathrm{C}-\mathrm{NMR}$ spectrum for $(\mathrm{C}=\mathrm{O})_{\text {cha }}$. 194.8, 192.6 and 191.5 for S2, S3 and S4 respectively ${ }^{17}$. Some of prepared compounds studding the 2D-NMR (HMBC) and we found for compound S2 three coupling for type ${ }^{1,3} J_{H, C}$ the first; between $\mathrm{C}^{\prime \prime}$ " to be the distance $\mathrm{d}=120 \mathrm{ppm}$ and proton $\mathrm{Hb} \mathrm{d}=7.5 \mathrm{ppm}$, and the second between $\mathrm{C} 1$ " for the distance $\mathrm{d}=124 \mathrm{ppm}$ and proton $\mathrm{Ha} \mathrm{d}=8$ ppm. Third of coupling ${ }^{1,3} \mathrm{~J}_{\mathrm{H}, \mathrm{C}}$ between $\mathrm{C}=\mathrm{O}$ for the distance $\mathrm{d}=195 \mathrm{ppm}$ and proton $\mathrm{Hb}$ for the distance $\delta=7.5 \mathrm{ppm}$. or the distance $\delta=195 \mathrm{ppm}$ and proton Ha for the distance $\delta=8 \mathrm{ppm}$.

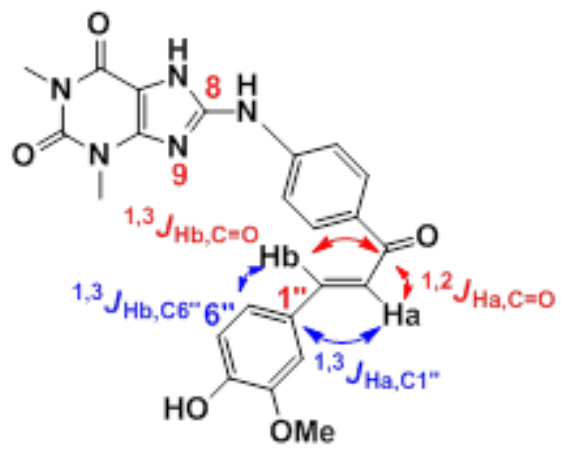

For S3 also studding the HMBC-NMR and we found three coupling for type ${ }^{1,3} J_{\mathrm{H}, \mathrm{C}}$ the first; between $\mathrm{C} 6$ " to be the distance $\mathrm{d}=115 \mathrm{ppm}$ and proton $\mathrm{Hb} \mathrm{d}=7.5$ ppm, and the second between $\mathrm{C} 1$ " for the distance $\mathrm{d}=135 \mathrm{ppm}$ and proton $\mathrm{Ha} \mathrm{d}=8 \mathrm{ppm}$. Third of coupling ${ }^{1,3} \mathrm{~J}_{\mathrm{H}, \mathrm{C}}$ between $\mathrm{C}=\mathrm{O}$ for the distance $\delta=194 \mathrm{ppm}$ and proton $\mathrm{Hb}$ for the distance $\delta=7.5$ ppm. The second type of coupling is ${ }^{1,2} J_{H, C}$ there are two coupling between $\mathrm{C}=\mathrm{O}$ for the distance $\delta=194$ ppm and proton $\mathrm{Ha}$ for the distance $\delta=8 \mathrm{ppm}$ and between $\mathrm{C} 4$ " for the distance $\delta=135 \mathrm{ppm}$ and proton 
H3" for the distance $d=7.3$ ppm. ${ }^{18,19}$.The second type of coupling is ${ }^{1,2} J_{\mathrm{H}, \mathrm{C}}$ there are one coupling between $\mathrm{C}=\mathrm{O}$ for the distance $\delta=195 \mathrm{ppm}$ and proton $\mathrm{Ha}$ for the distance $\delta=8 \mathrm{ppm}$. of chalcone (S2-S4) that reacted with thiourea in the presence ethanolic Sodium hydroxide to give corresponding. Pyrimidine as well as reacted with hydrazine in the presence absolute ethanol to give pyrazoline derivatives. Pyrimidine and pyrazoline can be

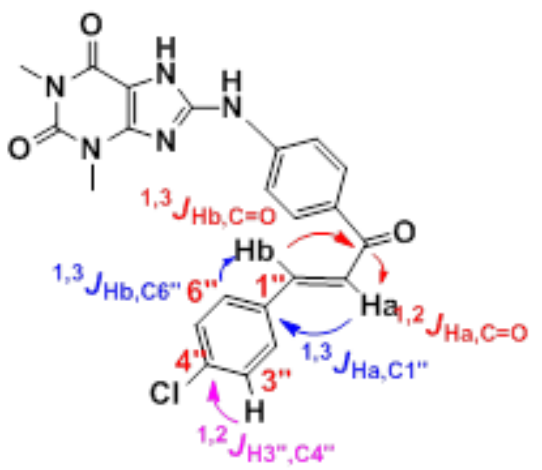

prepared by Scheme 3. ${ }^{1} \mathrm{H}-\mathrm{NMR}$ and ${ }^{13} \mathrm{C}-\mathrm{NMR}$ of Pyrimidine and pyrazoline compounds can be show in Table. 1 and 2 respectively.

\section{Biological part}

Control of microbial population is necessary to prevent show of disease, infection, decomposition, spoilage and contamination and caused by them. The newly synthesized compounds were screened for their antimicrobial activity in vitro against bacteria (Staphylococcus aureues, Escherichia coli) and fungal (Candida albicans) as show in Fig. 1. The antimicrobial activity results discovered that most of the tested compounds have moderate to strong activity. The most effective compounds are S3, S5 and S8 when these compounds were compared with the reference compounds (DMSO, distill water) we found that they have an antimicrobial activity higher or almost equal to them. While candida albicans not responsive for all compounds.

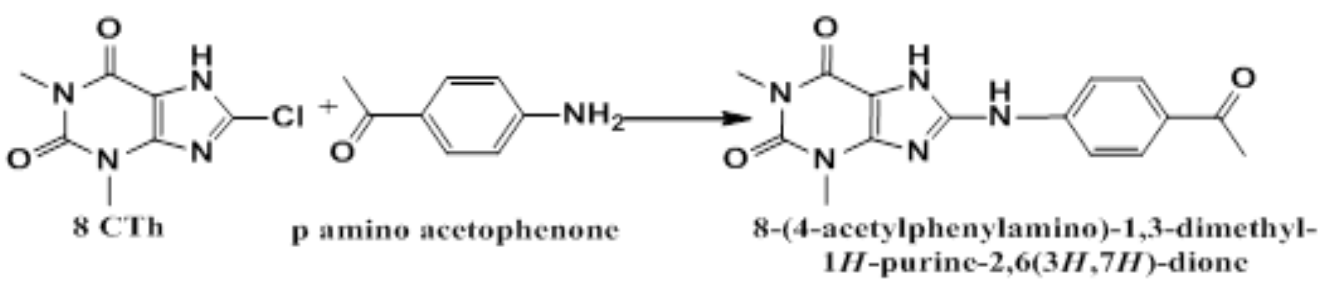

Scheme 1. Synthesis of 8-(4-acetyl phenyl amino)-1,3-dimethyl-1H-purine-2,6 $(3 H, 7 H)$-dione.<smiles>[R]c1ccc(/C=C/C(=O)c2ccc(Nc3nc4c([nH]3)c(=O)n(C)c(=O)n4C)cc2)cc1</smiles>

\begin{tabular}{l|r}
\multicolumn{1}{c|}{$\mathrm{R}$} & cha. \\
\hline $3-\mathrm{OMe}, 4-\mathrm{OH}$ & $\mathrm{S} 2$ \\
$4-\mathrm{Cl}$ & $\mathrm{S} 3$ \\
$4-\mathrm{NMe}_{2}$ & $\mathrm{~S} 4$
\end{tabular}

Scheme 2. Synthesis of chalcone 
Table. 1: Characteristic FTIR absorption, ${ }^{1} \mathrm{H}-\mathrm{NMR}$ and ${ }^{13} \mathrm{C}$-NMR bands of compounds (S5-S7)

\begin{tabular}{lccccccc}
\hline No. of comp. & \multicolumn{3}{c}{$\mathrm{IR}$} & \multicolumn{1}{c}{${ }^{1} \mathrm{H}-\mathrm{NMR}$} \\
\hline & $\mathrm{N}-\mathrm{H}_{\mathrm{py}}$ & $\mathrm{N}-\mathrm{H}_{\mathrm{py}}$ & $\mathrm{C}_{p y}$ & $\mathrm{C}_{\mathrm{py}}$ & $\mathrm{C}_{\mathrm{py}}$ & $\mathrm{C}_{\mathrm{py}}$ & $\mathrm{C5}_{\mathrm{py}}$ \\
S8 & 3218,3363 & 9.15 & 6.21 & 3.97 & 151.3 & 42.7 & 50.1 \\
S9 & 3346,3227 & 9.15 & 3.71 & 3.78 & 151.7 & 42.3 & 50 \\
S10 & 3223,3334 & 9.63 & 3.75 & 3.9 & 150.5 & 42.9 & 42.3 \\
\hline
\end{tabular}

Table. 2: Characteristic FTIR absorption, ${ }^{1} \mathrm{H}-\mathrm{NMR}$ and ${ }^{13 \mathrm{C}} \mathrm{C}$-NMR bands of compounds (S8-S10)

\begin{tabular}{lccccccccc}
\hline No.of comp. & \multicolumn{3}{c}{ IR } & \multicolumn{3}{c}{${ }^{1} \mathrm{H}-\mathrm{NMR}$} \\
& $\mathrm{S}-\mathrm{H}$ & $\mathrm{C}=\mathrm{C}$ pyr. & C=N pyr. & $\mathrm{N}-\mathrm{H}$ & $\mathrm{S}-\mathrm{H}$ & $\mathrm{C} 2 "$ & $\mathrm{C} 4 "$ & ${ }^{13}$ C-NMR & C6" \\
S5 & 2644 & 1648 & 1523 & 8.18 & 1.36 & 62.7 & 103.4 & 164.6 & 142.3 \\
S6 & 2653 & 1649 & 1523 & 8.71 & 1.41 & 65.4 & 102.9 & 159.4 & 142.9 \\
S7 & 2646 & 1652 & 1500 & 9.68 & 1.37 & 65.8 & 103.6 & 159.6 & 142.9 \\
\hline
\end{tabular}

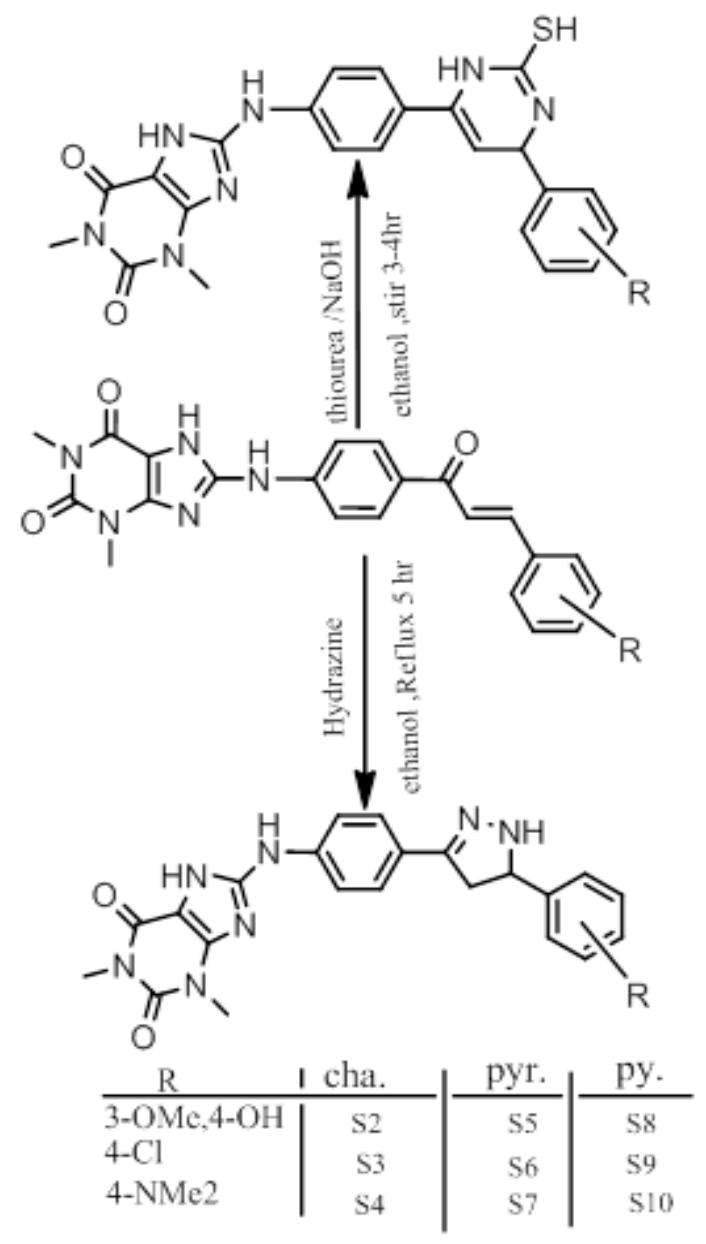



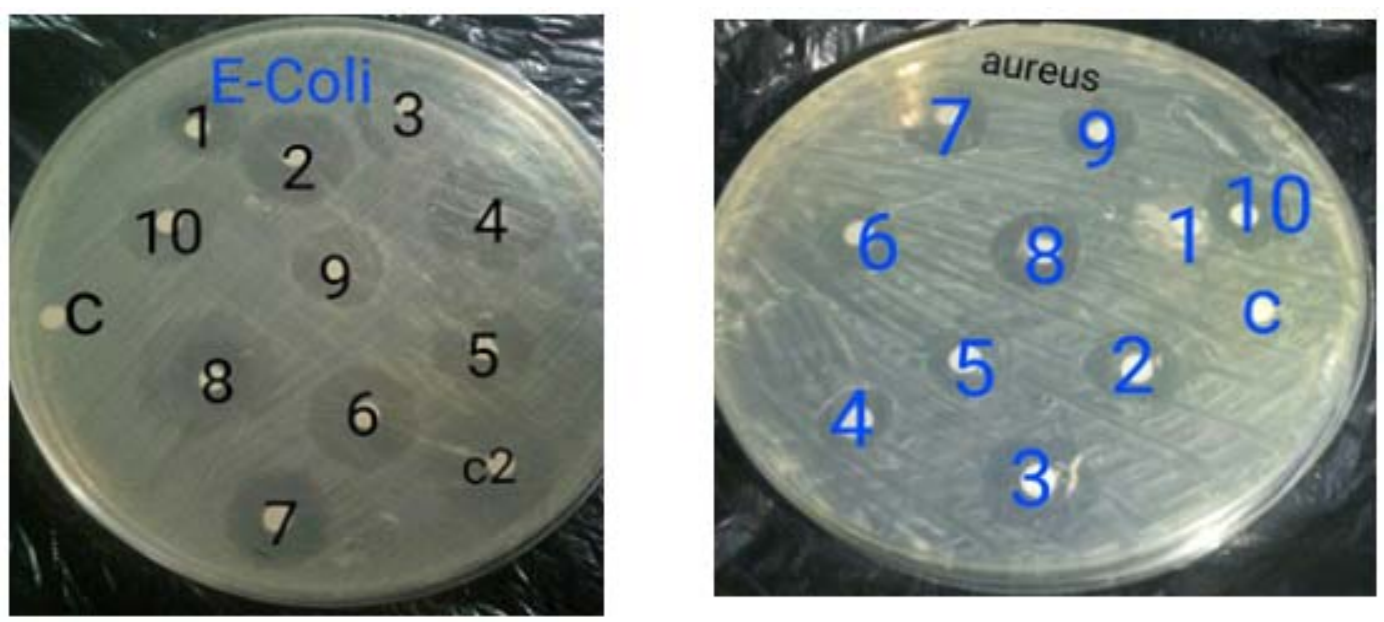

Fig. 1. Zone of inhibition of compounds against Staphylococcus aureues, Escherichia coli and Candida albican

Table. 3: Zone of inhibition measured in $\mathrm{mm}$ : no activity $(0.0)$, very weak activity $(<7$ $\mathrm{mm})$, weak activity $(7-10)$, moderate activity $(11-15 \mathrm{~mm})$, strong activity (>15 mm)

\begin{tabular}{cccc}
\hline No. of Comp. & $\begin{array}{c}\text { Gram-positive } \\
\text { S. sureus }\end{array}$ & $\begin{array}{c}\text { Gram-negative } \\
\text { E-coli }\end{array}$ & $\begin{array}{c}\text { Fungal } \\
\text { Candida ablicans }\end{array}$ \\
\hline S1 & 12 & 18 & - \\
S2 & 14 & 22 & - \\
S3 & 17 & 23 & - \\
S4 & 14 & 25 & - \\
S5 & 13 & 20 & - \\
S6 & 19 & 23 & - \\
S7 & 15 & 17 & - \\
S8 & 16 & 24 & - \\
S9 & 13 & 16 & - \\
S10 & 15 & 18 & - \\
Control/DMSO & 0 & 0 & 0 \\
Distill water & 0 & 0 & \\
& & &
\end{tabular}

\section{CONCLUSION}

In this study we are reported synthesis of many chalcone derivatives from 8-CTh. The work included preparation of chalcone compounds and then prepared pyrimidine when react with thiourea, in other hand react with hydrazine to prepared pyrazoline. These derivatives were found active angst bacterial and not response to fungal. These derivatives confirmed from spectral data analysis; FT-IR, ${ }^{1} \mathrm{HNMR},{ }^{13} \mathrm{CNMR}$ and 2D-NMR.

\section{ACKNOWLEDGMENT}

The authors are thankful prof. Dr. Najim A.AL-Masoudi, Chemistry Department, Konstanz University, Germany, for making and analyzer NMR spectra. Also we are really appreciating the help of Miss Zahra khthair, College of Education -Biology, AL-Qadisiya University. 


\section{REFERENCES}

1. Szentmiklosi, A. J; Cseppento, A; Gesztelyi, R; Zsuga, J; Körtvely, A; Harmati G; Nanasi P.P. Current Medicinal Chemistry., 2011,18, 3695-3706.

2. Barnes, P. Theophylline. Pharmaceuticals, 2010, 3, 725-747

3. Riksen, N.P.; Smits, P.; Rongen, G.A. The cardiovascular effects of methyl xanthines. Handb. Exp. Pharmacol., 2011, 200, 413-437.

4. Mosselhi, A .M; Abdallah, M. A; Metwally, N.H, El-Desoky I. A; Break, L. M. Arkat USA, Inc., 2009, 1551-7012, 53 -63.

5. Mamedov, I, G; Khrustalev, V.N; Bayramov, M.R; Maharrmov, A.M. indian J. Chem., 56,192-196.

6. Solankee, A; Tailor, R. Chemistry International 2016, 2 (4), 189-200.

7. Tawfiq, M. T; Zaier A. J. Journal of College of Basic Education., 2015, 21.18

8. Younis, F.M. College of Basic Education Researchers Journal., 2011, 11, 711-721.

9. Khazaal, M. S. Tomma, J. H. Ibn al- haitham J. For pure \& appl. Sci ., 2011, 24.2.

10. Hsieh, C.T. Bioorganic \& Medicinal Chemistry
Letters., 2012, 22, 3912-3915

11. Elarfi.M. J. Sci. Revs. Chem. Commun., 2012 22, 103-107.

12. Voskiene, A; Mickevicius, V; and Mikulskiene, G. Arkivoc .2007. $x v, 303-314$.

13. Abdalla, M.; Gomha, S; Abd el-aziz, M; Serag, N. Turk J Chem., 2016. 40 .441-453.

14. Dabholkar, V.V; Ansari, Y.F. Indian Journal of chemistry., 2008, 47, 1758-1761.

15. Joshi, V. D; Kshirsagar, M. D.; Singhal, S. International Journal of ChemTech Research., 2012, 4, 971-975.

16. AbdulReda N.A; Abass, A. K and Gebur, N. A. European Journal of Chemistry., 2016, 2 , 206212.

17. Savant, S. S, Patel, S.M, Gamit, E. A, HaJiyani, H. U, Patel, S. A, Patel, P. S and Patel, K.C. Int J Chem Sci., 2017, 15, 2, 139.

18. Abdul-Rida, N.A;Mohammed, T.I;AlMasoudi,N.A;Frotscher,M. Medical chemistry research., 2017, 22(19) Nakanishi,K; Mill Valley, California: University Science Books., 1990, 136. 